\title{
UCRL-JC-155308
}

LAWRENCE LIVERMORE NATIONAL LABORATORY

\section{Turbulence in the Divertor Region of Tokamak Edge Plasma}

M. V. Umansky, T. D. Rognlien, X. Q. Xu, R. H. Cohen, and W. M. Nevins

September 3, 2003

Plasma Edge Theory Conference, San Diego, California, September 3-5, 2003 


\section{DISCLAIMER}

This document was prepared as an account of work sponsored by an agency of the United States Government. Neither the United States Government nor the University of California nor any of their employees, makes any warranty, express or implied, or assumes any legal liability or responsibility for the accuracy, completeness, or usefulness of any information, apparatus, product, or process disclosed, or represents that its use would not infringe privately owned rights. Reference herein to any specific commercial product, process, or service by trade name, trademark, manufacturer, or otherwise, does not necessarily constitute or imply its endorsement, recommendation, or favoring by the United States Government or the University of California. The views and opinions of authors expressed herein do not necessarily state or reflect those of the United States Government or the University of California, and shall not be used for advertising or product endorsement purposes.

This is a preprint of a paper intended for publication in a journal or proceedings. Since changes may be made before publication, this preprint is made available with the understanding that it will not be cited or reproduced without the permission of the author.

This report has been reproduced directly from the best available copy.

Available electronically at http://www.doc.gov/bridge

Available for a processing fee to U.S. Department of Energy

And its contractors in paper from

U.S. Department of Energy

Office of Scientific and Technical Information

P.O. Box 62

Oak Ridge, TN 37831-0062

Telephone: (865) 576-8401

Facsimile: (865) 576-5728

E-mail: reports@adonis.osti.gov

Available for the sale to the public from

U.S. Department of Commerce

National Technical Information Service

5285 Port Royal Road

Springfield, VA 22161

Telephone: (800) 553-6847

Facsimile: (703) 605-6900

E-mail: orders@ntis.fedworld.gov

Online ordering: http:/ / www.ntis.gov/ordering.htm

\section{OR}

Lawrence Livermore National Laboratory

Technical Information Department's Digital Library

http: / / www.llnl.gov/tid/Library.html 


\title{
Turbulence in the Divertor Region of Tokamak Edge Plasma
}

\author{
M.V.Umansky *, T.D.Rognlien, X.Q.Xu, R.H.Cohen, and W.M.Nevins \\ Lawrence Livermore National Laboratory, Livermore, CA 94550, USA
}

\begin{abstract}
Key words edge plasma, turbulence simulations
PACS 53.35.Ra, 52.65.Kj, 52.40.Hf

Results of recent modeling of tokamak edge plasma with the turbulence code BOUT are presented. In previous studies with BOUT the background profiles of plasma density and temperature were set as flux surface functions. However in the divertor region of a tokamak the temperature is typically lower and density is higher than those at the mid-plane. To account for this in the present study a poloidal variation of background plasma density and temperature is included to provide a more realistic model. For poloidally uniform profiles of the background plasma the calculated turbulence amplitude peaks near outer mid-plane, while in the divertor region the amplitude is small. However, present simulations show that as the background plasma profiles become more poloidally non-uniform the amplitude of density fluctuations, $\tilde{n}_{i}$, starts peaking in the divertor. It is found that in the divertor region the amplitude of $n_{i}$ fluctuations grows approximately linearly with the local density of the background plasma, $n_{i 0}$, while the amplitude of $T_{e}$ and $\phi$ fluctuations is positively correlated with the the local electron temperature, $T_{e 0}$. Correlation analysis shows that plasma turbulence is isolated by the x-points.
\end{abstract}

\section{Introduction}

It is generally accepted that the global confinement in a tokamak is strongly affected by the conditions at the edge. Plasma turbulence, and the resulting anomalous cross-field plasma transport, are crucial physical processes in the boundary region. This makes edge plasma turbulence a major issue for tokamak physics. In recent years there has been a substantial progress in numerical simulations of edge plasma turbulence with the BOUT code [1]. BOUT is an electromagnetic 3-D turbulence code solving in a realistic geometry of a divertor tokamak a system of Braginskii-like [2] fluid equations for plasma density, $n_{i}$, electron and ion temperatures, $T_{e, i}$, electron and ion parallel velocity, $V_{\| e, i}$, electrostatic potential, $\phi$, and the vector potential, $A_{\|}$. The basic physics model and geometry of BOUT are described in $[1,3]$. BOUT has demonstrated encouraging similarity to experimental spatial and temporal spectra of edge plasma fluctuations in DIII-D [4], C-Mod [5] and NSTX [6].

Typically, in BOUT simulations the magnetic geometry is obtained from the magnetohydrodynamic (MHD) equilibrium code EFIT [7], and the profiles of the background plasma density, $n_{i 0}$, and temperature, $T_{e, i 0}$, are based on fits to experimental data, or on simulations by the tokamak edge transport code UEDGE [8].

In previous studies with BOUT $[3,4,9,10]$ the poloidal variation of background plasma parameters was not taken into account. This poloidal variation is usually caused by the boundary conditions at the divertor plate, and results in plasma temperature decreasing and plasma density growing towards the plate. For the high-recycling regime the ratio of conditions at the mid-plane and near the divertor plate, $T_{m i d} / T_{d i v} \sim n_{d i v} / n_{m i d}$, is on the order of 3-10, and more, so the poloidal variation may be substantial [11]. In the present study we analyze the effects of using poloidally dependent profiles for the background plasma for turbulence simulations with BOUT.

\section{Methodology}

We use the magnetic geometry based on a typical DIII-D magnetic reconstruction from EFIT. The geometry is shown schematically in Fig. 1

\footnotetext{
* Corresponding author: e-mail: umansky@llnl.gov, Phone: +1-925 422 6041, Fax: +1-925 4233484
} 


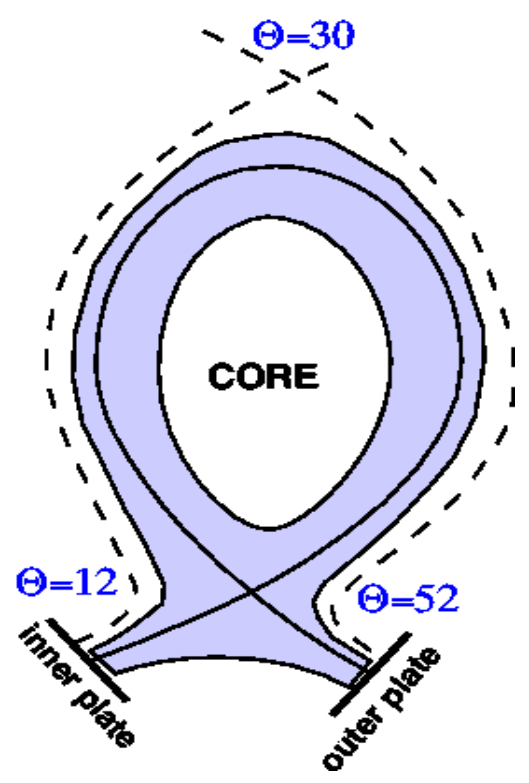

Fig. 1 Computational domain geometry in the radial-poloidal plane. The poloidal index, $\theta$, runs from the inner plate to the outer one. Poloidal locations of x-points are also shown.

For the present study BOUT is used in the mode when only fluctuating quantities with zero toroidal average are allowed. Thus the background average profiles for the background plasma density, $n_{i 0}$, and temperature, $T_{e, i 0}$ are "frozen".

For the background plasma we use a set of simulated toroidally-symmetric profiles which qualitatively and quantitatively approximate the equilibrium state of the edge plasma as known from experimental data and UEDGE simulations. The radial profiles for $n_{i 0}$ and $T_{e, i 0}$ at the mid-plane are taken as a typical fit to DIII-D data. The poloidal profiles of $n_{i 0}$ and $T_{e, i 0}$ were chosen to cover a range of variation. For the poloidal variation of $T_{e 0}$ we use the analytic expression

$$
T_{e 0}(s)=\left[T_{d i v}^{\gamma}+4 s(1-s)\left(T_{m i d}^{\gamma}-T_{d i v}^{\gamma}\right)\right]^{1 / \gamma} .
$$

Here $\mathrm{s}$ is the poloidal coordinate measured from the inner plate to the outer one and normalized to unity, $T_{d i v}$ is the divertor temperature, $T_{m i d}$ is the mid-plane temperature, and constant factor $\gamma$ was typically chosen around 2-3.

The ion temperature, $T_{i}$, was set equal to $T_{e} / 3^{1}$, and the poloidal variation of $n_{i 0}$ was then set to keep $n_{i 0}\left(T_{e 0}+T_{i 0}\right)$ constant along the field line. The plasma flow velocity, $V_{i|| 0}$, was set zero, and the DC electric potential was set zero.

We have analyzed a set of five BOUT cases with same radial profiles of $n_{i 0}, T_{e, i 0}$ upstream but different conditions in the divertor:

Table 1 Approximate parameters at separatrix at the mid-plane and in the divertor for five BOUT cases

\begin{tabular}{|c|c|c|c|c|}
\hline Case & $T_{e 0, \text { mid }}, \mathrm{eV}$ & $T_{e 0, \text { div }}, \mathrm{eV}$ & $n_{i 0, \text { mid }}, \times 10^{14} \mathrm{~cm}^{-3}$ & $n_{i 0, \text { div }} \times 10^{14} \mathrm{~cm}^{-3}$ \\
\hline 1 & 50. & 50. & 1.2 & 1.2 \\
\hline 2 & 50. & 25. & 1.2 & 2.5 \\
\hline 3 & 50. & 20. & 1.2 & 3.3 \\
\hline 4 & 50. & 12. & 1.2 & 4.8 \\
\hline 5 & 50. & 7. & 1.2 & 9.0 \\
\hline
\end{tabular}

\footnotetext{
1 The scaling factor $1 / 3$ was applied to these and most of the previous BOUT simulations for the reasons of numerical stability
} 


\section{Simulation results}

\subsection{Local scaling}

For all five analyzed cases BOUT was run to the state of saturated turbulence. Then the root-mean-square (RMS) amplitudes of the saturated turbulence were analyzed. The RMS amplitudes are shown in the radial-poloidal index plane for case 1 in Fig. 2.
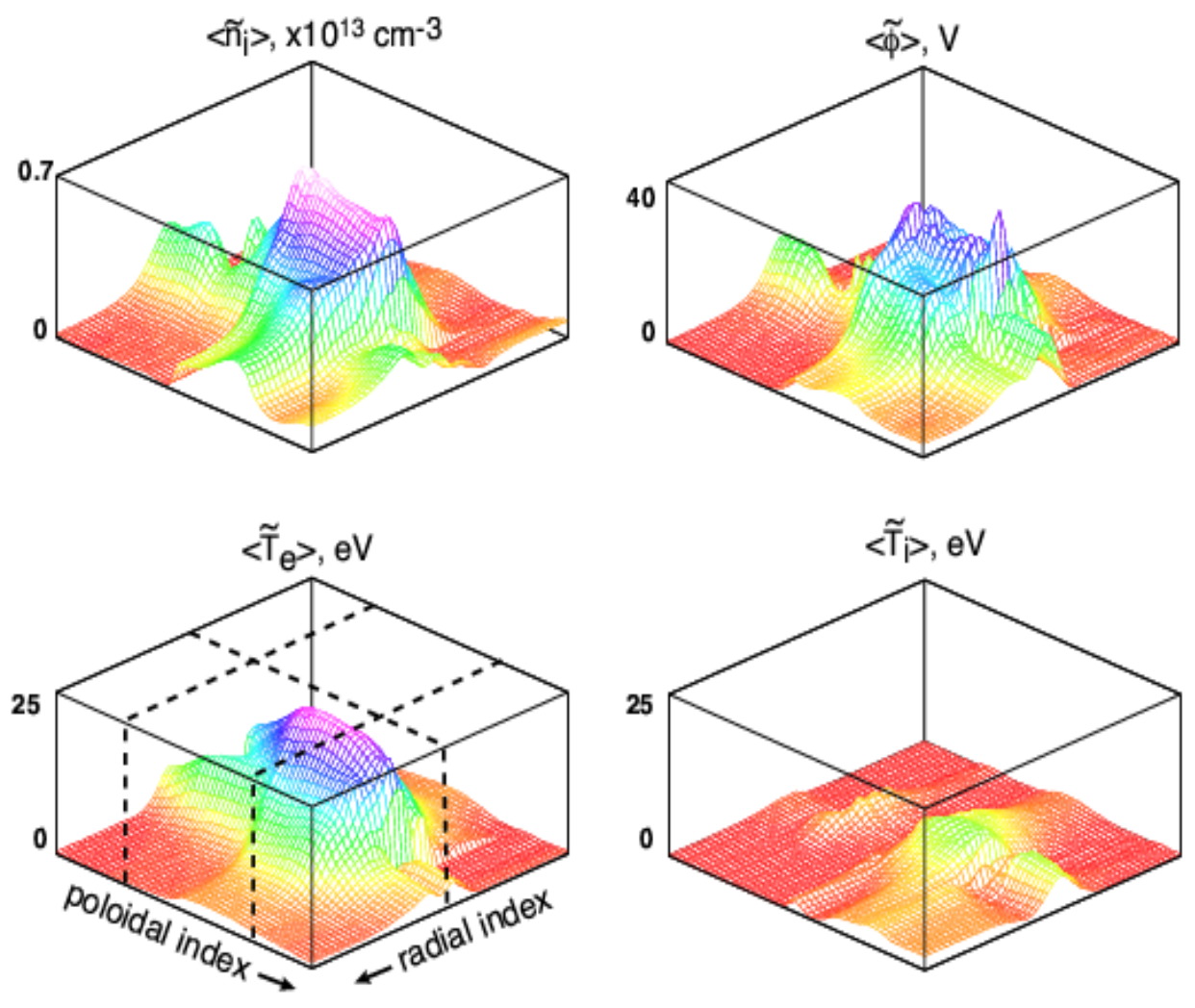

Fig. 2 Fluctuation RMS amplitudes for case 1, poloidally uniform profiles. The dashed lines show the locations of separatrix and $\mathrm{x}$-points.

The RMS amplitudes for case 3 are shown in Fig. 3.

Comparing Figs. 2 and 3 one can note that for poloidally uniform profiles (case 1) the fluctuation amplitudes peak at the mid-plane. On the other hand, for poloidally non-uniform profiles (case 3 ) there is a peak of $\tilde{n}_{i}$ fluctuations near the outer divertor plate. The amplitude of the divertor $\tilde{n}_{i}$ fluctuations appears to be roughly proportional to the local plasma density, $n_{i 0}$, for both inner and outer divertor (see Fig. 4). Thus the larger $\tilde{n}_{i}$ amplitude in the divertor can be attributed to the larger $n_{i 0}$ there.

Similar to the correlation between the $\tilde{n}_{i}$ amplitude and the local $n_{i 0}$ it was found that fluctuation amplitudes of $\tilde{T}_{e}$ and $\tilde{\phi}$ in the divertor are correlated with the local $T_{e 0}$. As the temperature in the divertor is lower than upstream, the absolute values of $\left\langle\tilde{T}_{e}\right\rangle$ and $\langle\tilde{\phi}>$ are lower in the divertor.

The amplitude of fluctuations upstream were found to be insensitive to the variation of parameters in the divertor. This raises the question whether the upstream turbulence is uncoupled from the divertor turbulence. 

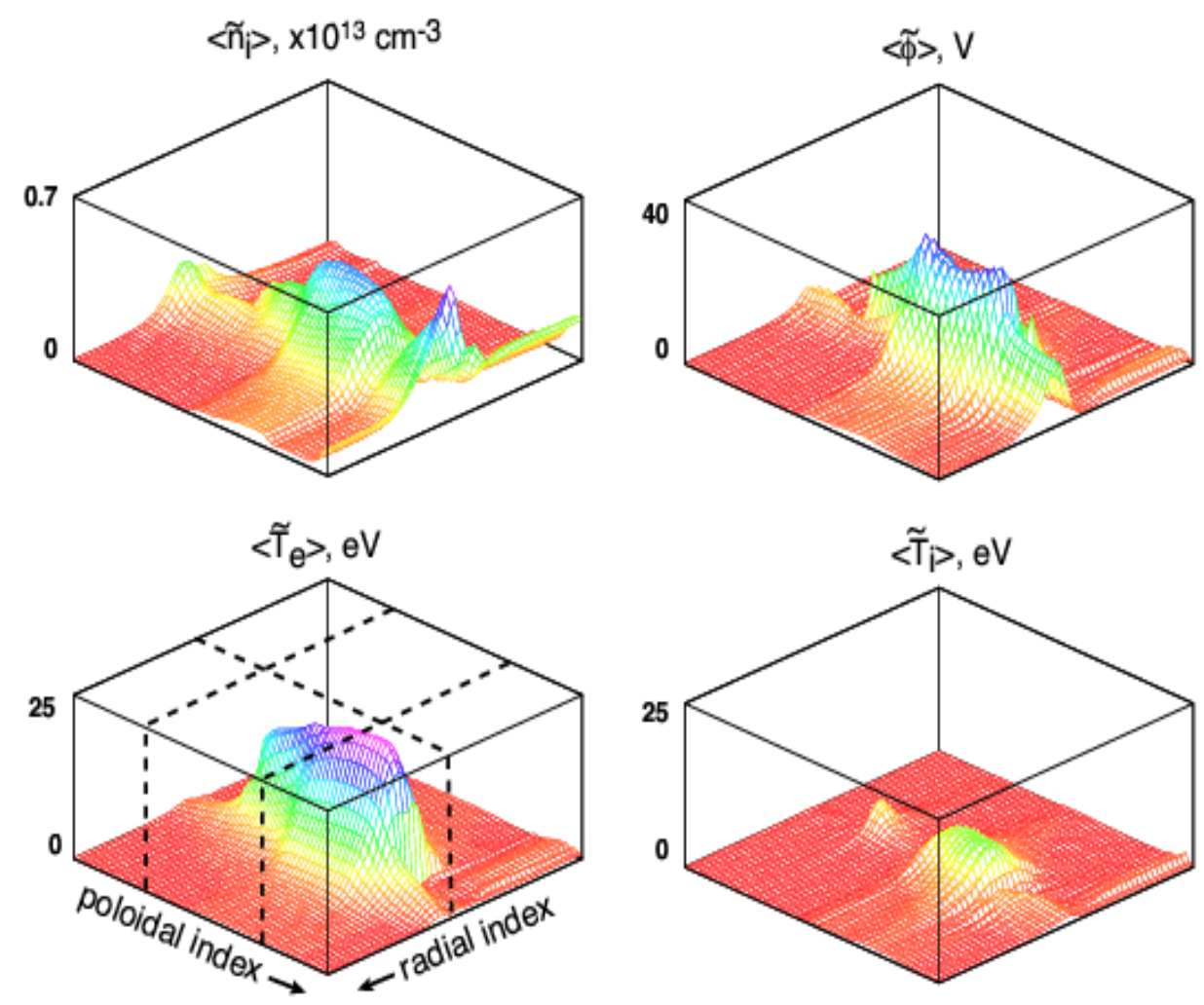

Fig. 3 Fluctuation RMS amplitudes for case 3, poloidally non-uniform profiles.
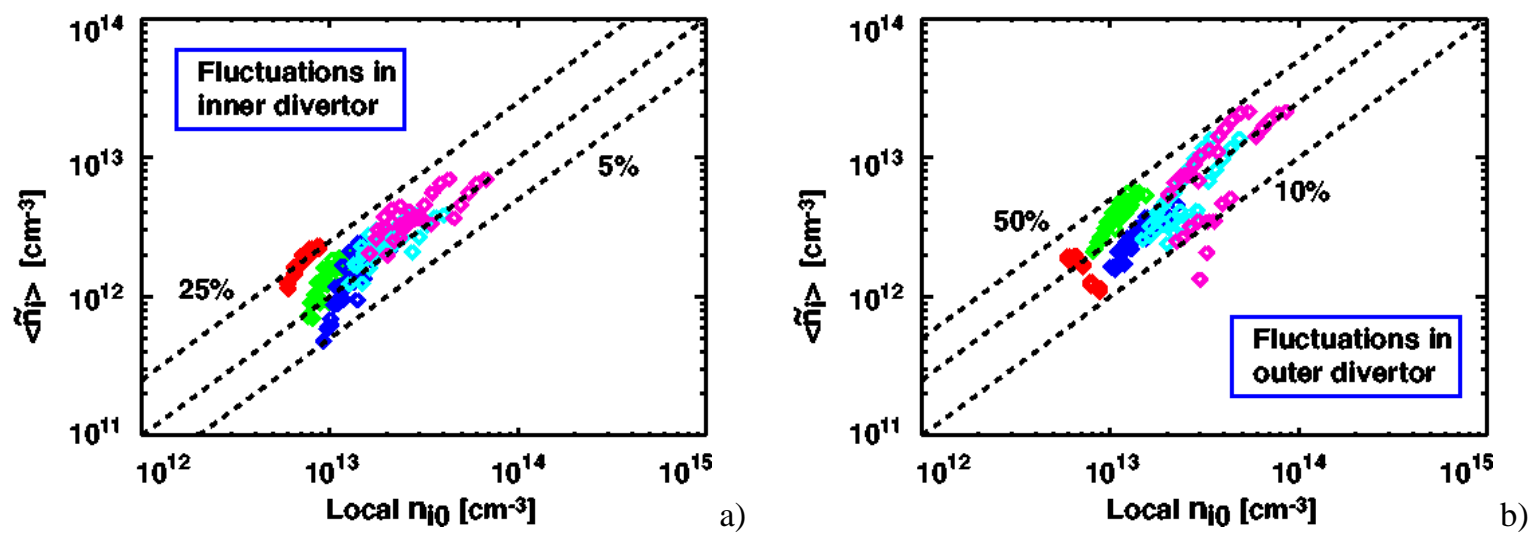

Fig. 4 Correlation of the RMS fluctuation amplitude $<\tilde{n}_{i}>$ with the local $n_{i 0}$ for the inner (a) and outer (b) plate regions.

\subsection{Correlation analysis}

We use the data analysis package GKV [12] to calculate the cross-correlation function for the turbulent potential. The cross-correlation function is defined as follows: 


$$
C(\tilde{\phi}, \tilde{\phi})=\frac{<\tilde{\phi}\left(r_{0}, \theta, \xi+\delta \xi, t+\tau\right) \tilde{\phi}\left(r_{0}, \theta_{r e f}, \xi, t\right)>_{\xi, t}}{<\left|\tilde{\phi}\left(r_{0}, \theta_{r e f}, \xi, t\right)\right|^{2}>_{\xi, t}}
$$

Here $\xi$ is the toroidal grid index, $\theta$ is the poloidal index, $\tau$ is the time lag, $\theta_{\text {ref }}$ is the reference poloidal index.

For case 3 , for a flux surface right outside of the separatrix, with mid-plane $\theta_{\text {ref }}=38$ and $\tau=0$, the calculated cross-correlation function is shown in Fig. 5

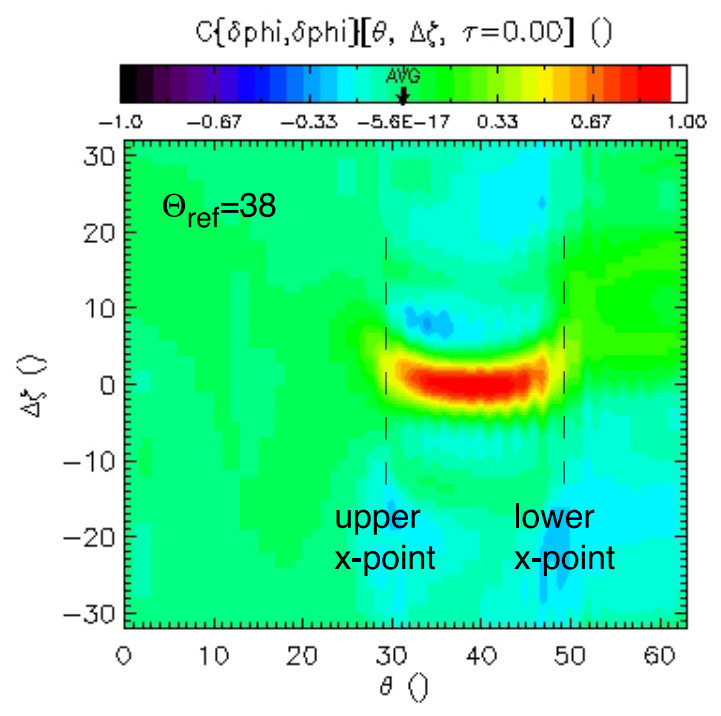

Fig. 5 Cross-correlation function for the simulated turbulence. The toroidal coordinate $\xi$ is also a magnetic line label here, so $\delta \xi=0$ means the point are on the same magnetic line.

Figure 5 shows that the cross-correlation has cutoffs at both the lower x-point and the upper x-point. Thus the turbulence appears to be disconnected by the $\mathrm{x}$-points which is consistent with previous results from BOUT [9].

\section{Discussion}

One of our results is that the normalized fluctuation amplitudes in the divertor: $\tilde{n}_{i} / n_{i 0}, \tilde{T}_{e} / T_{e 0}$, and $\tilde{\phi} / T_{e 0}$ appear to be approximately constant over the range of variation of $n_{i 0}$ and $T_{e 0}$. This is consistent with the probe measurements in DIII-D edge indicating that the normalized potential fluctuation amplitude, $e \tilde{\phi} / T_{e}$, in the divertor is comparable to that at the outer mid-plane [13].

Partitioning of the turbulence by the x-points from our correlation analysis is consistent with previous computational results from BOUT [9] and the theoretical arguments that the strong shearing of the magnetic field near the x-point leads to uncoupling of the perturbations in the upstream scrape-off layer from those below the $\mathrm{x}$-point [14]. It is interesting that even the upper $\mathrm{x}$-point which is rather remote from our computational domain appears to have a strong effect.

During the linear stage the divertor turbulence appears to be growing outside of the separatrix in the outer divertor, and inside of the separatrix in the inner divertor. As these locations are the regions of unfavorable curvature, this observation apparently points to the ballooning nature of the driving mechanism. An important question that requires further analysis is the role of the boundary conditions at the target plate. Identification of the driving mechanism of the divertor turbulence is the subject of ongoing analysis. 


\section{Summary and conclusions}

We have conducted analysis of turbulence simulations with the BOUT code using poloidally non-uniform poloidal profiles for the background plasma that mimic higher density and lower temperature in the divertor chamber. It was found that as the background plasma density, $n_{i 0}$, becomes larger in the divertor, the density fluctuation amplitude $\tilde{n}_{i}$ becomes larger. The amplitude of the density fluctuation normalized by the background local density, $\tilde{n}_{i} / n_{i 0}$, appears to be approximately constant in the divertor, at the level of 10-20\%. Similarly, there is a positive correlation between the $\tilde{T}_{e}$, and $\tilde{\phi}$ fluctuation amplitudes, and the local electron temperature, $T_{e 0}$. The turbulence amplitude upstream appears to be insensitive to the variation of the divertor conditions. Correlation analysis shows that the upstream turbulence is uncoupled from the divertor turbulence which is consistent with the strong shearing of the magnetic field near the x-points.

Acknowledgements We thank D.D.Ryutov for helpful discussions. This work is performed for USDOE by Univ. Calif. LLNL under contract W-7405- ENG-48 and was supported as LLNL LDRD project 03-ERD-099.

\section{References}

[1] X. Q. Xu and R.H.Cohen, Contr. Plas. Phys. 36158 (1998).

[2] S.I. Braginskii, Transport processes in a plasma, Reviews of Plasma Physics, Vol. 1, Ed. M.A. Leontovich (Consultants Bureau, New York, 1965), p. 205.

[3] X.Q. Xu et al., Phys. Plasmas 71951 (2000).

[4] X. Q. Xu et al., J. Nucl. Mater. 266-269 993 (1999).

[5] A. Mazurenko et al., Phys. Rev. Lett. 89 (22) (2002).

[6] X.Q. Xu et al., New Journal of Physics 4 53.1-53.15 (2002).

[7] L.L.Lao et al., Nucl. Fusion 251611 (1985).

[8] T.D.Rognlien et al., Contr. Plas. Phys. 34362 (1994).

[9] X. Q. Xu et al., Nucl. Fusion 40731 (2000).

[10] X. Q. Xu et al., Nucl. Fusion 4221 (2002).

[11] P.C. Stangeby, "The Plasma Boundary of Magnetic Fusion Devices" Bristol, Institute of Physics Publishing, 2000.

[12] W.M.Nevins, "GKV User's Manual" (unpublished).

[13] R. A. Moyer et al., J. Nucl. Mater. 241-243 633 (1997).

[14] D. Farina et al., Nucl. Fusion 331315 (1993). 